\title{
Who are We, and Who (or What) Do We Want to Become? An Evolutionary Perspective on Biotransformative Technologies
}

\author{
James Lyons-Weiler ${ }^{1}$ (D)
}

Received: 7 April 2021 / Accepted: 13 July 2021 / Published online: 16 September 2021

(c) The Author(s) 2021

\begin{abstract}
Human evolution sits at several important thresholds. In organic evolution, interplay between exogenous environmental and genetic factors rendered new phenotypes at rates limited by genetic variation. The interplay took place on adaptive fitness landscapes determined by correspondence of genetic and environmental relationships. Human evolution involved important emergences that altered the adaptive landscape: language, writing, organized societies, science, and the internet. These endogenous factors ushered in transformative periods leading to more rapidly evolving emergences. I explore the impact of development of emerging biotransformative technologies capable of being applied to effect self-genetic modification and artificial intelligence-augmented cognition on the evolutionary landscape of phenotypes important to cognitive plasticity. Interaction effects will yield unanticipated emergences resulting in hyperrealm adaptive landscapes with more rapid evolutionary processes that feed back upon more fundamental levels while vastly outpacing organic evolution. Emerging technologies exist that are likely to impact the evolution of cognitive plasticity in humans in ways and at rates that will lead to societal upheaval. I show that the theoretical contribution of organic evolution in future human evolution is expected to become comparatively insignificant relative to that made by endogenous environmental factors such as external cognition aids and manipulation of the human genome. The results support the conclusion of a strong recommendation of a moratorium on the adoption of any technology capable of completely altering the course of human evolution.
\end{abstract}

Keywords Cognitive plasticity $\cdot$ Evolutionary theory $\cdot$ External cognitive aids $\cdot$ Genetic manipulation $\cdot$ Human evolution

\section{Introduction}

During the course of human evolution, evolutionary milestones, or critical emergences, are suspected of having occurred. Somewhere in the span of vertebrate evolution, for example, a type of consciousness emerged that allowed retention and retrieval of important details about the environment. This would have changed the adaptive landscape of those organisms, who could now evolve adaptive behavioral strategies. Thus, cognitive plasticity originated, which, in turn, brought forward new platforms upon which evolutionary processes could play out. These platforms, or "evolutionary hyperrealms" such as thought, consciousness, adaptive learning, language, culture, and technology, exist one level "above" the level upon which they originate. These new

James Lyons-Weiler

jim@ipaknowledge.org

1 The Institute for Pure and Applied Knowledge, Pittsburgh, PA, USA realms have increasingly allowed these animals to change their external environments. Changes in the environment are the mainstay of evolutionary understanding of new sources and types of selective pressures, and thus the emergence of new replicating features that compete with each other for survival and reproduction can also create feedback loops to lower realms.

Especially with the evolution of cognitive plasticity (CP), the potential for the transmission of information among members of the same species emerged via demonstrated behavior, learning, and language. This included the evolution of the capacity of species for intragenerational transmission of information among individuals, and between generations. Somewhere in time during the evolution of hominins, the cognitive abilities for language and to represent complex and accurate descriptions of the environment and re-creation of (symbolic representation of) historical events and relationships among entities emerged. This critical emergence then created a new adaptive landscape in a hyperrealm above the physical landscape, allowing the formation of and 
competition among symbolic representations of the physical world. Evolution in the new hyperrealm, which Henriques (2019) considers a real "metaphysical," includes development of competing cultures, societal norms and mores, and introducing a new type of environmental influence that feeds back on the genetics of humans. Evolution in the cultural hyperrealm itself has had its own critical emergences, such as the transitions into and through agriculture, the creation of government, the printing press and books (fixed symbolic externalized human memory), the development of science and mathematics, the formalization of education, and the development and emergence of democracy.

Environmental factors that have, and could in the future influence human evolution, fall into three broad categories: organic (those that came with this planet), endogenous (those that we create), and exogenous (those that are created by things we create). These can be typed usefully into five "kinds," as laid out in Table 1. The primary focus of this essay is on the future, and therefore will primarily consider the third, fourth, and fifth kind of environmental factors.

The pace of evolutionary lability of culture is much faster than organic evolution. The emergent hyperrealms of evolution are not independent and in fact interact; our organic environment of course has also been radically changed by technology, and environmental toxins are an increasing source of morbidity and mortality in the world. Our individual experiences have entered a new major shift in the virtual realm; we have a world of information at our fingertips, as conditions that foster evolution are present in this virtual hyperrealm: the competition among ideas and facts, all driven by actions of humans with vestiges of our tribal ancestors. The opportunity for rapid consumption of information leads to an informal educational framework in which profound challenges to strongly held worldviews can be just a click away. The very act of being confronted with opposing views in a species in which, evolutionarily

Table 1 Types of environmental factors that have and could influence human evolution

\begin{tabular}{|c|c|c|}
\hline Kind & E type & Examples \\
\hline First kind & Organic & $\begin{array}{l}\text { Nature, the elements, climate, resources } \\
\text { Lewontin-type environmental modification } \\
\text { Epigenetic }\end{array}$ \\
\hline Second & Endogenous & $\begin{array}{l}\text { Language } \\
\text { Writing } \\
\text { Culture } \\
\text { Civilization } \\
\text { Pollution, mutagens }\end{array}$ \\
\hline Third & Endogenous & Genetic, self-genomic modification \\
\hline Fourth & Endogenous & Machine-based human augmentation \\
\hline Fifth & Exogenous & $\begin{array}{l}\text { AI-programmed AI, AI-genetic modifica- } \\
\text { tion, extermination }\end{array}$ \\
\hline
\end{tabular}

speaking, members are "used to" dealing with conspecifics in small group dynamics leads to the emergences of groups of persons whose affiliation was entirely unlikely prior to the development of the internet. The most recent hyperrealm is social media platforms where, from any location, any individual or group can contact millions. We have crossed the threshold, and there is no turning back.

At present, we stand on the brink of two additional precipices which could radically alter our evolutionary path. There are two critical thresholds I will explore formally and about which I argue we must make a moral choice, as a species, and as individuals, whether we "should" step through. The first of these is the ability of humans to reprogram their own genome via the development of new genetic modification technologies that, in principle, could lead to direct feedback from the brains of humans to the very genome encoding of those brains, a cycle that is unprecedented in the evolutionary history of consciousness on our planet. The second is the emergence of technologies that could augment our memories (external storage devices) and expand human intelligence.

The approach I take is a heuristic mathematical comparison of limits implied by these factors. The conclusions are written in the context of a time of great uncertainty and social strife, and I therefore reserve the right to adapt and change positions if these emergences materialize.

\section{Cognitive Plasticity as an "Increasingly Complex" Trait}

The usual treatment of the evolution of cognitive plasticity considers the evolution of $\mathrm{CP}$ in vertebrates leading to Homo sapiens, and their descendants. However, it is clear that invertebrate relatives of vertebrates have adaptive and flexible condition-specific behaviors, no matter how simple, and these phenotypes qualify as candidates for CP.

Starfish, for example, which have actual focused-image vision (Petie et al. 2016), can distinguish between healthy and ailing sea urchins while feeding. Sea urchins themselves change their feeding behavior in response to resource density and risk of predation (Zhadan and Vashenko 2019). The complexity of feeding and mating behavior of insects and crustaceans implies a degree of cognitive plasticity; bees change their nectar foraging behavior (Shackelton et al. 2016), even changing their communication dancing with hive-mates in order to share information on nectar quality (DeMarco and Menzel 2005; De Marco 2006).

In terms of cognitive plasticity in vertebrates, fish would likely have small, but nonzero genetic $\times$ environment $(G x E)$ interaction terms relevant to $\mathrm{CP}$, in spite of clearly having exhibited learning, and even tool use (see "Einstein of the Sea"; Balcombe et al. 2016). Reptiles similarly would seem 
to have small, but nonzero $G x E$ terms relevant to $\mathrm{CP}$, showing evidence of spatial learning (Noble et al. 2012) as well as retaining memory of solutions to complex feeding tasks, demonstrating evidence of visual discrimination, reversal learning, judging equivalence, finding novel ways to access food, and play behavior, and potentially awareness of gaze direction (Wilkinson and Huber 2011). A recent study in crows (Neider et al. 2020) provides evidence of both primary and secondary subjective experience, and histological studies of various avian species' brains show mammal-like structural arrangement of the cerebral cortex, implying that consciousness does not require the complex mammalian-like layering of the cerebral cortex (Stacho et al. 2020).

Complex traits are those that are influenced by multiple genes, and usually have phenotypes that are non-discrete and can be impacted by environmental factors. Due to intentional programming of intergenerational learning, cognitive plasticity can be seen as an increasingly complex trait. For the sake of our consideration, we will avoid the complex but useful formal representation of polygenic traits by Dudbridge (2013) and leave, for the sake of clarity of exposition, our environmental terms expanded. The current exploration is also heuristic.

\section{Organic Emergences (Exogenous)}

Organic evolution is understood to begin with an influx of random mutations in a population, contributing to genetic variation. The input of new genetic variation is considered to be a potential limiting factor on the rate of evolution. New genetic variation arises from two sources: de novo mutations, and migration of individuals carrying new variation into a population. Combined, these two factors are classically considered to place a gross upper limit on the rate of evolution.

Epistatic interactions, however, are defined by relationships among genes, and the role of regulatory reprogramming of biological pathways as unleashing potentially "hidden" potential influences of genetic variation. The disruption of coevolved and co-regulated genetic modules via genetic modification - including unintended off-target modification and epigenetic consequences - may uncover phenotypic variation beyond that which may be implied by raw total genetic variation (Le Rouzic and Carlborg 2008).

It is impossible to know the full effect of any new genetic variation on survival and reproduction - even if one specific phenotype is known to correspond to a given genotype. The impact of technological environmental factors leading to increased survivorship of alleles that have in the past been lethal or sublethal has led to fairly pessimistic expected outcomes (e.g., Lynch 2016); however, such an analysis places the rate of technological advancement at a slower pace than genetic drift of otherwise deleterious alleles, which is false for any individual allele but for the full set of otherwise deleterious alleles, no doubt a local eventuality that would lead to perceived need for ever-increased rates of genetic manipulations.

Any variation related to differential survival and reproduction in an interbreeding population will shift over time, favoring alleles that contribute positively to fitness in the context of the environment in which the organism lives. Organisms and their phenotypes evolve on adaptive landscapes defined by the relationship between individual fitness-related phenotypes and the environment. On adaptive landscapes, populations can be seen as traveling through time along fitness gradients as gene frequencies related to relevant corresponding phenotypes shift in response to environmental selection pressures (Wright 1984). It is understood that mathematically, most allele frequency changes that occur over time are not, or are only weakly related to fitness (Ohta 1973, 2002; Kimura 1983). This leaves the most interesting alterations related to Darwinian evolution to those changes that are related to fitness, and to the study of gene-gene interactions and the interplay between variation in gene interaction networks, gene birth and death processes (Nei and Rooney 2005), and gene-environment interactions $(G x E)$ as important considerations of realms of basic and applied organic evolutionary inquiry.

The traditional empirical approach to $G x E$ research is to first identify a statistically significant interaction between a genetic variant and an environmental measure, and then to proceed to characterize the interaction based on further criteria. This has been described extensively by Schlichting and Pigliucci (1998). Additional clarifications by Pigliucci (2005) on three considerations are worth reviewing.

In the current explorations, I adopt a population genetics model of additive gene and environmental factors with known $G x E$ interactions. Therefore, we are not trying to find or show statistical significance; the current treatment is relevant only for traits where interactions exist between genetic and environmental factors of interest, and in the case of endogenous $E$ 's, this is tautologically necessary. Specifically, without the myriad genetic abilities to carry out the cognitive operations necessary to learn how to conduct genetic self-modification studies, our species would not be able to engender endogenous environmental factors that influence $\mathrm{CP}$.

At a more fundamental consideration, our phenotype of interest is cognitive plastic phenotype (CP), given genetic $(G)$ and environmental factors $(E)$, the typical model for studying phenotypic plasticity,

$C P=G+E+G \times E$

In this notation, the $G \times E$ term is designated as that part of the phenotype that exists due exclusively to the interaction 
between the relevant, specific genetic variation and environmental variation. $G$ is not a specific, individual gene but is instead that portion of the variation in the specific phenotype of interest that is heritable; $E$ is that portion that is influenced directly and exclusively by the environment. Thus, $G x E$ can represent the interaction between single genetic and environmental factors, or among a single and multivariate factor, or among multivariate factors. The covariance matrices among variables describe the strength and direction of their influences, and these, importantly, are expected to show cascading changes with the emergence of each new hyperrealm. That is, the covariance matrices among traits and the genes they encode are never truly fixed; with modification and externalization, they can become increasingly decoupled, or more rigidly determined.

This model allows the empirical study of the limits of reaction norms to detect whether - and in the context of information on specific genes, how - genetics and environment interact to influence a given phenotype of interest. In application, empirical limitations include the requirement of large sample sizes to characterize empirically the biological and statistical significance of $G x E$, the complexity of considering polygenic traits, and lack of reproducibility of studies (especially in psychiatry; see a review by Assary et al. (2018) that includes these limitations); as clarified, however, our consideration is theoretical and heuristic, not empirical.

If we define $E$ of the first kind as the environment as nature presents itself - external to our species - the effect is a direct correspondence of the influence of environment on survival and reproduction determined, in part, by genetics. For all of organic evolution, $E$ has been, to a large extent, $E$ of the first kind: environment as nature presents itself, modified in form for camouflage, mate attraction, and shelter, reflecting cognition that may or may not involve biological (cognitive) computations beyond instinct.

Behavior that leads to modification of $E$ of the first kind organisms also modify their environment by their presence, or by their behavior, thus changing or altering their own adaptive landscape, thus influencing their own evolutionary trajectory (Lewontin 1978). Integrated with genetics is epigenetics, a layer of semi-heritable regulation that has its own rate of evolution, with faster turnover than genomic evolution, feeding back upon genetic evolution by helping to define the adaptive landscape. Beltran et al. (2020) provide a first-ever estimate of the rate of heritable epigenetic mutations (epimutations) in C. elegans and estimate that while heritable epimutations occur at a rate that is an order of magnitude $(25 \times)$ greater than the rate of (baseline) genetic mutations, they noted that most epimutations last on the order of 2-3 generations. This low persistence of variation likely reflects species' adaptability in a dynamic environment. Fluctuations and variation in absolute rates and types of mutations appearing in the population exist over geographical space, and over time (e.g., Harris and Pritchard 2017).

The role of epigenetics in the current consideration of rates of human evolution given technological impacts is unclear; transgenerational epigenetic modifications in mammals seems commonly limited to $2-3$ generations, with methylation patterns reestablished via germ line chromosomal packaging and often overwritten via recombination.

In this exploration, the phenotype of interest is human cognitive plasticity specifically because this is the very origin of de novo forms of endogenous environmental factors and will very likely continue to be a high-priority target of such factors. Running a human brain requires the expression of a massive proportion of the human genome and is associated with more alternative splicing than gene expression in any other organ. How these spliceoforms relate to cognitive plasticity is uncertain. At the same time, our collective cognitive processes have led to an increasingly mutagenic environment via widespread use of likely mutagenic pesticides in agriculture, food packaging, and dyes, and myriad tens of thousands of de novo compounds with unknown biological effects.

Lewontin-type environmental change involves feedback that specifically exacts cognition-derived influences on the adaptive landscape that influences a species' survival and reproduction in a manner that does not specifically then further impact cognition phenotypes. In the current treatment, they are considered environmental factors of the first kind. They contrast, for example, with the emergence of formal education. Formal education is an example of an emergent phenotype that results in intergenerational transmission of abilities to arrange the environment which then feeds back as an influence upon the populations' cognition. The point is not to debate whether cognitive activities actually go beyond instinct in humans, but rather to address the superpositioning of evolving systems that evolve at a higher realm but that arose from within a more slowly evolving system of vertical transmission. Similar complex interactions may be seen in resource use, population density, and productivity in seed beetles (Burls et al. 2014). This demonstrates, for the purpose of this exploration, the consideration of the evolution of human cognitive plasticity and consciousness. The models to be explored can be used to describe equally well a theory of emergences with, and without, self-awareness of the type we might call human conscious awareness. 


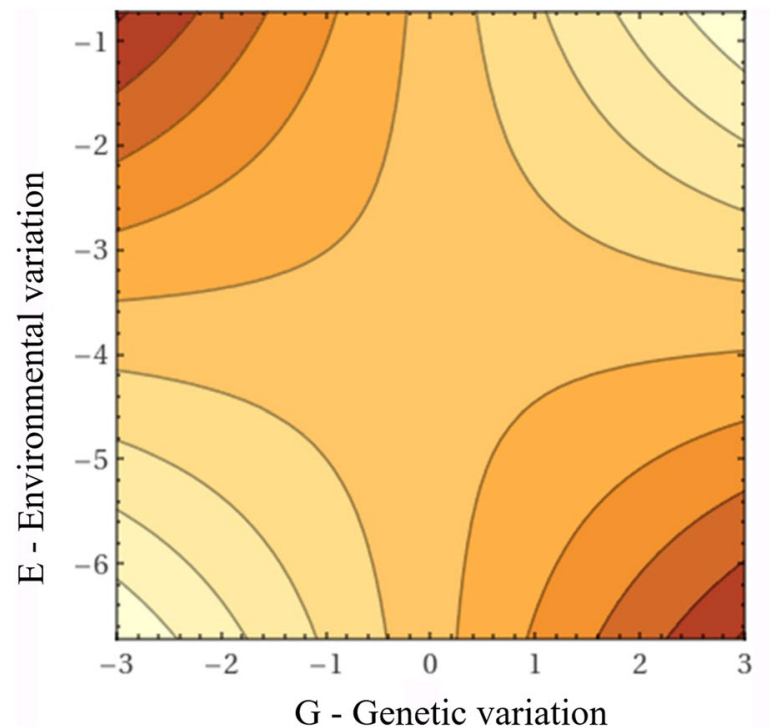

$\uparrow Z=$ Phenotypic variation response or fitness of same

Fig. 1 Adaptive landscape portraying variation in fitness involving interaction between genetic and environmental variation. $G$ genetic variation; $E$ environmental variation; $Z$ represents a measure of outcome in the specific form of evolution (fitness) and more generally outcome functions (e.g., fitness, replication, persistence, duration, adoption by others)

\section{Self-Selection Emergences (Endogenous): Phenotypes of Endogenous Origins}

Due to the self-referential impact of the mind influencing itself, hominins with significant cultural impact on survival and reproduction transitioned to

$C P=G+E+G \times E_{1}+G \times E_{2}$

where the organic environmental factors of the first kind $\mathrm{E}_{1}$ (the direct impacts of influences external to a species) and environmental factors of the second kind, i.e., the selfreferential environmental impact $\mathrm{E}_{2}$ on $\mathrm{G}$, are distinct in form and yet interact. Thus emerge the first phenotypes of endogenous origins (PEOs): characteristics of cognition that were adaptive but that originated out of de novo cognition on the part of the carrier of genes endowing the capacity for such valuable cognitive feats.

The function (Fig. 1) provides expectation of a curved (nonlinear) "phenotypic landscape," which would set the stage for the emergence of conditional, environmentally dependent norms of reaction in which either of the two factors $(G$ or $E$ ) are more likely to influence the outcome of an environmental variable $(Y)$ on a $\mathrm{CP}(Z)$ with an evolutionarily stable binary condition reaction norm likely. The portion of the solution space is arbitrary, and the meaning of $G$ and its signed value an abstraction; it is sufficient to see the interaction influencing the third axis $(Z=C P)$ as a complex landscape. Since this is a function of phenotype it is proper to see $\mathrm{CP}$ as a feature expressed by and thus subject to feedback from selection. This particular phenotypic landscape of possibilities would allow selection toward the preservation of any genetic variation contributing to dichotomous conditional behaviors, provided such genetic variation presented itself through mutation.

An example of an evolutionarily stable strategy might be the teaching of conditional hunting strategies. Consider, for example, the time invested in learning a new conditional rule to use a particular behavioral tactic while hunting. Given how success translates into increased relative survival and reproduction, the rate of evolution would of course still be limited by $G$ with the conservation of flexible traits being the dominant selective force against the loss of an environmentally adaptive CP.

This could help explain the cultural lag commonly seen in the face of clear signals requiring the development of new strategies, and the annoying habit of humans to commit the Concorde fallacy and continue to invest in losing schemes long after clear and painful evidence that the optimality of a strategy no longer fits the empirical data.

This possibility includes all advances to date in culture and could be considered the evolutionary basis for value systems favoring tradition. Resistance to change could similarly be explained: once concerted and genuine efforts have been made to establish a process, such as that required to formalize education, resistance to reform and institutional momentum are difficult to overcome. This consideration excludes, of course, any of the historic and damnable eugenics programs, because they directly impact (or could impact) $G$.

While it is worth contemplating the potential relevance of societal struggles between progressive and conservative viewpoints, it is hazardous to contemplate a genetic basis for such viewpoints, given that direct experience in the superpositioning of adaptive flexibility of positions on topics can lead to changes in viewpoints in both directions. Again, the current analysis is heuristic, and behavioral exceptions to the expectation would be the exception.

\section{Directed Genetic Modification (Endogenous) and Phenotypes of Endogenous Origin}

With advances in genetic modification, any technologybased attempt to improve cognitive traits directly would lead to a distinctly novel set of endogenously generated environmental factors, i.e., environmental factors of the third kind $\left(E_{3}\right)$, and would also (literally) create new genomic components $\left(G_{2}\right)$. These endogenously generated environmental 
factors are first-in-mankind and stand apart as a profound change in the history of organic evolution.

For clarification on $G_{2}$ as a concept, this includes genetic modification (additions and subtractions that result from genetic modification technologies applied to heritable genetic variation). However, $G_{x}$ could also apply to additional capacities from cultural abilities that derive from human genetic propensity to, for example, develop and use external cognitive devices, addition of cells from other species during embryogenesis (chimeras), or alteration of neurodevelopment via biohacking. Thus, numerous endogenous environmental factors would beget (at a minimum) their own potential for genetic and environmental interactions.

As a result, CP quickly runs away with add-on technologies as, for example,

$$
C P=G+E+G \times E_{1}+G \times E_{2}+\left(G_{2} \times E_{3}\right)+\left(G_{m} \times E_{n}\right)
$$

We will expand on other exogeneous environmental factors in the next section. Staying focused on genetic modification only for now, with a new or impacted part of the genome, and $E_{3}$ representing acts of genetic modification (heritable or not), the potential for conditions for emergences increases dramatically. Since genetic modification adds new genes intentionally (or subtracts them), we can discuss the $C P$ addome: that portion of the genome that influences cognitive plasticity that is introduced not via random mutation but instead via directed genomic manipulation, and $\left\{G-G_{2}\right\}$ would be the $C P$ subtractome. Both changes would represent willful and intentional shifts of the parts of the genome that encodes the brain (or physiological factors that influence the central nervous system), making these changes endogenous even though the technology used is external. Such influences would lead to PEOs.

\section{Augmented Consciousness}

We already store records of memories in digital and print form and have done so since the earliest writing (Cross 1980). Etchings of pottery shards in Ban Po village from ca. 4500-3750 BCE in China are stylistic precursors to later Chinese writing systems; the emergence of writing had a profound effect on cultural evolution in China (Ebrey 2010). The spread of the early Canaanite writing via the Phoenicians throughout the Mediterranean had a significant impact on economics. The writings of each generation of course then contributed to successive generations' understanding of the past. Being a symbolic representation of reality, writing systems are themselves hyperrealms of external storage of human cognition.

Artificial intelligence is a cultural game changer. We now stand at the threshold of new technology for transmitting information into and out of the human brain. Noninvasive and invasive technologies currently exist. The US Army, for example, is exploring the use of noninvasive transcranial magnetic stimulation as "human-technology systems," the technology of which is expected to impact the perceptions, decisions, and actions of soldiers. Invasive technology such as Elon Musk's Neuralink allow mind-originated wireless control over external technologies; it is unknown if the technology eventually will allow the mind to receive external data.

In addition to the influences of genetic manipulations, technological leaps toward external memory devices might be envisioned, leading eventually to augmented consciousness on external devices via neuromorphic chips. The chips might be used as a solicited engagement (pinging) or via real-time two-way engagement with the human brain. Details of the gating of the flow of information, say, solicited versus real-time return, are significant and urgent questions, including the obvious and more basic ethical question of whether we, as a species, should engage in the communion of technology and biology.

If we do, the same model of lower limits applies for technology as for genetics.

But, if we embark on both journeys simultaneously, then the model becomes:

$$
\begin{aligned}
C P= & G+E+\left(G \times E_{1}\right)+\left(G \times E_{2}\right)+\left(G_{2} \times E_{3}\right) \\
& +\left(G_{3} \times E_{4}\right)+\left(G_{2} \times E_{3}\right) \times\left(G_{3} \times E_{4}\right)
\end{aligned}
$$

Defining a response limit as the limit of the range of evolution of $\mathrm{CP}$, the bounding the rate. The four response limits implied by the four equations are provided in Fig. 2.

At the core of this model is the question of whether our genetics generates the capacity for technology, and then in the self-referential manner, imbues us with the capacity for self-modification. I chose CP to focus on because it sits at the junction of biology and technology; "enhanced" CP will very likely lead to enhanced technology, and a runaway feedback is to be expected. As a lower limit, the minimum rate of divergence ignores the shift of the rate of cognitive evolution to the limit of Moore's law and the computer/brain baud rate. Empirically we find that since the minimum augmented $C P=5 G^{2}+2 G$, which contains no variation that requires the invocation of any organic $E_{x}$ term, will always eventually, for any fixed value of $E_{x}$ and with sufficient self-tinkering, see the technological evolution of $C P=5 G^{2}+2 G$ outpace the organic evolution of $C P=G+E+(G x E)$ at a runaway rate. The rate of evolution of cognitive plasticity will be increased manifold with new emerging means of self-modification of heritable (genetic or cultural) modifications. The crux of any moral question lies at this point: if this is just a continuation of the history of human self-modification, albeit at an increased rate, or something altogether new (especially due 


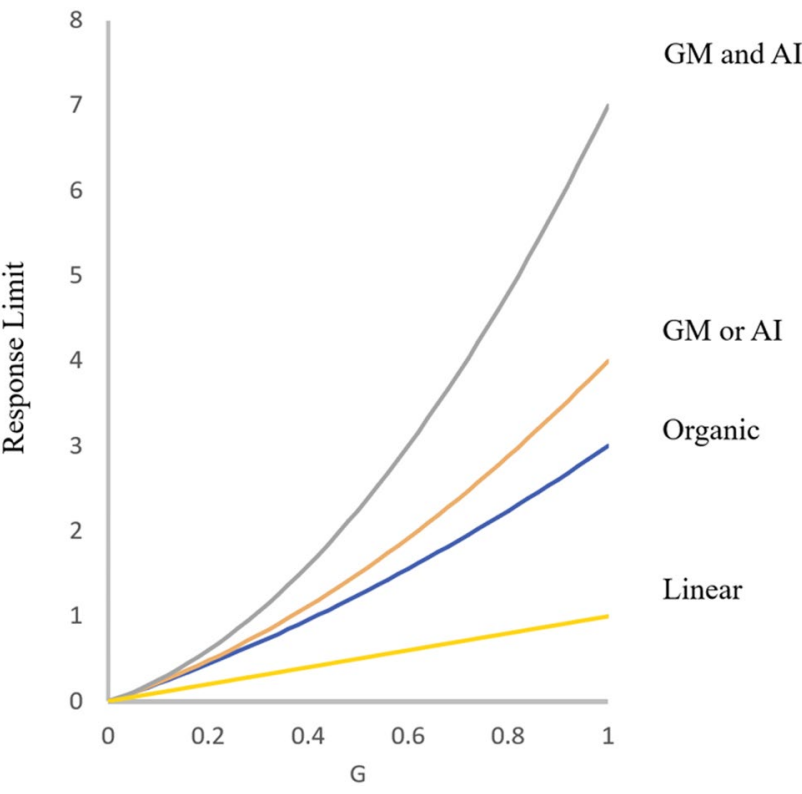

Fig. 2 Four response limit curves (positive solutions only) representing possible futures with a linear function for comparison. Each of these curves is of course an $n$-dimensional topographic manifold, which is difficult to represent in two dimensions. An important assumption is that exogenous environmental conditions are the same in all scenarios; changes in environmental scale of impact across scenarios invalidate comparisons. Because $G_{2}$ is endogenously rendered by $E_{3}$, the variation introduced (ultimately liability) is genetic; therefore, in the parameter span of $E_{3}$, it is proper to state it as variation in $G_{2}$. There is therefore replacement of $E_{3}$ by $G_{2}$, and thus $E_{3}$ falls out of the polynomial description. As a result, the polynomial of the positive solution space for the combined GM and AI model (and any two-factor endogenous environment model) is described by a lower limit of $C P=5 G^{2}+2 G$, reflecting that the endogenous environmental factors are, at their core, made possible by our genetics. With increasing genomic manipulation and reliance on external cognition leading to increased instances of inherited de novo genetic variation, an overwhelming influence of phenotypes of endogenous origin (due to the exponent on $G$ ) results. This emergence heralds the effective end of the importance of organic biological evolution in humans (due to the irrelevance or redundancy of $\mathrm{E}$ (exogenous environment) to describe the relationship). The same relative function placements persist for all $E_{x}>0$; also see Appendix

to the truly de novo $G_{x}$ 's) - and will provide fodder for future discussions and debate.

This simplistic representation leaves out overt consideration of the importance of the $\mathrm{P}, \mathrm{G}, \mathrm{E}$ variance and their covariances. At time $t_{0}$, prior to an emergence, there is coherence between the established relationships with (by definition) no significant variation among the entities of the nascent hyperrealm. Once an endogenous emergence is underway, the introduction of significance among the relationships can place new importance on existing $\mathrm{P}$, or $\mathrm{G}$, or, in the case of genetic modification (GM), introduce or remove $\mathrm{G}$ relevant to $\mathrm{P}$, altering the covariance matrices. In the domestication of animals, the overwhelming force of artificial selection leads to genetic costs (see Moyers et al. (2018) for numerous examples and counterexamples).

As with all things in nature, that which persists, exists; a subset of lineages of modified humans with disrupted covariance matrices will survive. In more concrete terms, the overriding influences of the commoditization of artificial manipulation of ourselves will lead to greater numbers of other-context deleterious alleles accumulating; this no doubt will lead to runaway use and dependency on genetic modification and high phenotypic variance once de novo addomic and subtractomic fitness components combine as synthetics find each other and reproduce.

Propagation of such alterations through genetic regulatory networks can be expected to alter other relationships among other phenotypes. Off-target effects are a significant source of concern; shifts in gene regulation that lead to changes that are indirectly related to the self-targeted phenotype, leading, at time $t_{l}$, to decoherence. This disruption of existing coadapted gene regulatory complexes then results in conditions ripe for rapid evolution in a manner much faster than would otherwise be possible. This can be visualized as rapid evolution through hills on adaptive landscape $L l$ by tunneling made possible via travel across hyperrealm adaptive landscapes $L 2, L 3$, or ( $L 2 x L 3)$ (see Fig. 3 for a conceptualization). This model explains in part how populations can move through fitness valleys across a Wrightian fitness landscape considering fitness components of a single trait; other traits can move the population through a lower fitness trough to a higher, previously unrealized fitness peak.

\section{Evolving at a Pace Set by Moore's Law}

In addition to the "technology $\times$ biology" $(t x b)$ communion implied by implants and computational explant cognitive devices, additional increase in the occurrence of $E$ of the fourth kind are expected, specifically (and importantly) due to advances in hardware and software evolving on their own adaptive landscape. If they impact human evolution, they are considered environmental factors of the fifth kind. While at first an endogenous environmental factor, if unleashed, this external influence could become effectively completely exogenous, if, for example, we unleash artificial intelligences (AIs) to design themselves, setting them upon their own adaptive landscapes that feed back upon our own. However, for the sake of this initial exploration, we leave the provenance of the liability of the original source intact (humans) and consider them endogenous. Developments otherwise could be environmental influences of the sixth kind (tech-designed tech and tech-designed software). 


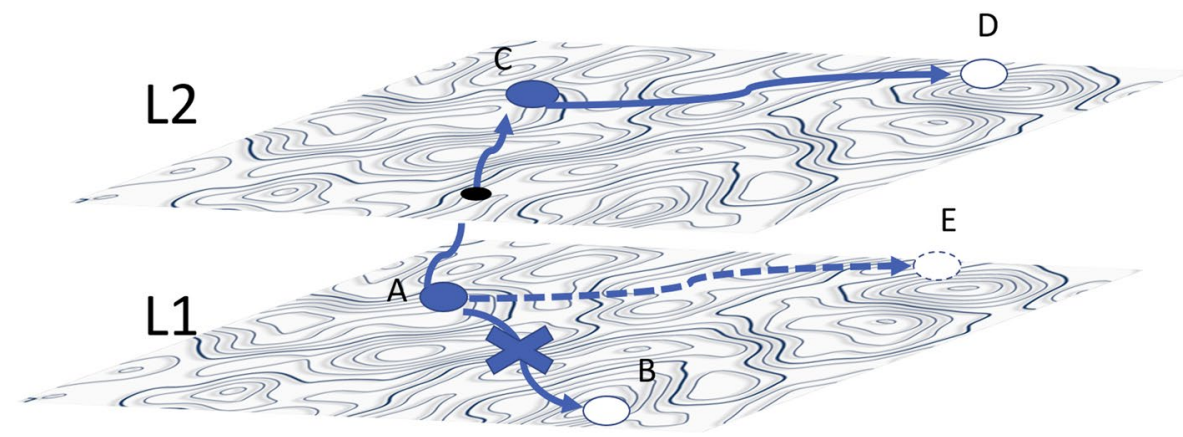

Fig. 3 Wrightian conceptualization of evolution at a fundamental level $(\mathrm{Ll})$ impeded $(\mathrm{A} \longrightarrow \mathrm{B})$ by troughs in the adaptive landscape, caused by insufficient or incorrect variation or environment unmatched to variation, alleviated or made irrelevant by the emergence of a new hyperrealm adaptive landscape (L2) upon which more rapid evolution is made with corresponding impact via fitness

\section{The "Golly-Gee-Whiz" Novel Tech Factor and Productivity Enhancement Demand}

It is clear that much of the public has no qualms about acquiring newly developed technology that might enhance their cognition and potential consciousness. An example is the "Neurophone efficiency optimizer," which claims to use ultrasound to feed patterns into the brain; a fundraiser to kick off the production of this version of Neurophone raised $\$ 1.5$ million in 2015; the associated website ${ }^{1}$ reports "over 5000 satisfied customers" although the inventor, portrayed as a perfectionist, is waiting for an improvement in his health to work on whatever unspecified shortcomings of the Neurophone might be preventing current sales.

The outcome of this fundraiser reveals that many individuals seeking advantage will adopt new self-improving technology, merely to keep a competitive edge, with little awareness of the fact that such individual decisions to produce and adopt such technology forever changes the phenotypic landscape upon which selection might work. It seems reasonable to expect that parents would want to be able to save their infants from inborn errors of metabolism. Does the subjective nature of "errors," defined by human cognition and culture, then automatically extend to the expectation that all parents might want to inject their infants with retrovirally induced insertion of "genius genes"? Which individuals who watched their parents suffer Parkinson's disease, or amyotrophic lateral sclerosis (ALS, Lou Gehrig's disease), or cancers with heritable risk would not petition to be allowed to undergo genetic modification to prevent, or alleviate, their fate of suffering?

\footnotetext{
$\overline{1}$ https://www.indiegogo.com/projects/neo-neural-efficiency-optim izer-neurophone\#/
}

tunneling on $\mathrm{L} 1(\mathrm{~A} \longrightarrow \mathrm{E})$. Multiple emergences, e.g., L3, LA, are conceived as layers stacked upon each other, each with more rapid evolution than the last, the cumulative effect of which can make the fundamentally slower, improbable adaption less relevant and potentially trivial. The disconnect between $E$ and $L 1$ caused by the $L 2$ emergence would also alter $L 1$

A necessary scholarly - and societal - question worth considering is: without cultural evolution anticipating the morality and ethics of AI-based and genetic self-modification, do we really know the full attendant consequences of what we are doing? Would a net average increase in IQ by 30 points translate into a net positive or negative for society? Perhaps all we can say is that we can expect that the variances in the extremes of $\mathrm{CP}$ related to intelligence would become larger as well.

\section{Runaway Emergences - Evolutionary Cascades - Are the Real Threat}

This analysis suggests that due to compounding changes on the cognitive phenotypic landscape, such adoptions will lead inexorably to additional new ideas and ways to "hack" ourselves, leading to opportunities for an emergence of runaway selection toward cognitive phenotypes that we may not yet even have terms to describe. Indirect effects on ourselves of unknown type and variety are also likely. For example, CRISPR shows great promise to allow the excision of segments of DNA from the human genome. In 2018, He Jiankui, a professor at the Southern University of Science and Technology, shocked the world and horrified the scientific community when he reported that he and two colleagues successfully conducted embryonic genome manipulation on twin sisters "Lulu" and "Nana" (pseudonyms) (Greely 2019). Dr. He had used CRISPR to alter a gene encoding the $\mathrm{C}-\mathrm{C}$ chemokine receptor type 5 (CCR5) protein, which HIV uses as a portal to enter healthy cells. The research team's reported goal was to demonstrate the technical feasibility of conferring immunity to HIV. As a result of this embryonic genome editing research, He and two other scientists were arrested and jailed by the Chinese government. 
There has been speculation about a goal of augmentation, given a potential functional role of CCR5 in human intelligence. The CCR5 protein is also thought to limit cognition. In 2016, Zhou et al. found that mice with nonworking CCR5 genes had lower cortical plasticity and hippocampal learning and memory (Zhou et al. 2016). And there may be medical incentive to further block CCR5; Joy et al. (2019) found that mice with a loss-of-function mutation of CCR5 had a faster recovery from stroke, including greater recovery of neurological impairments and cognitive function. Whether ulterior motives existed for targeting the CCR5 gene in humans is speculative.

There has also been speculation that if the information about the heritable genome editing conducted by $\mathrm{He}$ and colleagues had come through the peer review process, instead of through interviews and internet videos, the technique may have been seen as acceptable. If so, this reveals a degree of shortsightedness about the attendant consequences of heritable genome improvements. The runaway increase in this type of diverse addome variation is limited only by the imagination - in terms of black-market access to valuable gametes, breeding programs, and eugenicists bent on breeding a "superior" race of humans. Even without that overt goal, an otherwise random leap through the threshold will lead, generation after generation, to new interactions among PEOs; the multiplicative terms in Eq. 3 feed back on every generation in new ways, causing diverse PEOs to emerge. Here we see how Van Valen's Red Queen (1973) meets capitalist market demand; one can imagine what it would require to merely survive in a world of superhumans in which profits from genetic upgrades would be reinvested in better upgrades.

Coadapted gene complexes are genetic loci that together have become fused in their functional relationships underpinning complex traits in a manner that keeps the entire suite of genetic loci fixed in a population. The concept originated with Wallace (1953) and was championed by Theodosius Dobzhansky in 1970 as a mechanism of maintaining species delineations (Dobzhansky 1970). It is clear from our understanding that genetic manipulations have off-target effects that are the result of physical manipulation of the human genome. These would likely lead to disruption of normal coadapted gene complexes - not only at an unusual pace, but also leading to unusual functional pathways with evolutionarily arbitrary nonspecific effects happening in ever-increasing frequencies in the genome across the population.

As an increasingly diverse number of new options for genetic manipulation are invented, their effects on the human genome will combine in the future as the progeny of people with different addomes procreate. An explosion of phenotypes would result as regulatory networks become unraveled via off-target indirect genetic and epigenetic regulatory changes - and we would do well to recall that mutation rates are the primary limiting factor of evolution. The significance of genetic variation is compounded for complex multi-locus traits (Zhang et al. 2011). Thus, the specific and nonspecific effects of heritable and nonheritable genetic manipulations would, like all underlying genetic variation, be compounding for complex traits targeted by on- and offtarget modification of the human genome. It is appropriate in this context to be concerned about a multitiered version of the Bateson-Dobzhansky-Muller effect (Orr 1996) in which differences at the genetic and cultural level create degrees of reproductive isolation.

The consequences of projected rate of change in $C P$ over time due to shifts in the genetic walkover and through the hills and valleys of Wright's adaptive landscape, are, from a standpoint of evolution, terrifying.

\section{Society Begins to Respond}

In 2019, the World Health Organization (WHO) created a registry for all human genome-editing research, after calling for a halt to all such research pending further ethical inquiries (WHO 2019).

In September 2020, the US National Academy of Sciences (NAS) issued a report recommending restrictions on embryonic editing, including heritable human genome editing. The first in the series of restrictive recommendations offered was that:

No attempt to establish a pregnancy with a human embryo that has undergone genome editing should proceed unless and until it has been clearly established that it is possible to efficiently and reliably make precise genomic changes without undesired changes in human embryos. These criteria have not yet been met and further research and review would be necessary to meet them. (NAS 2020, p. 3)

\section{Societal Upheaval}

The NAS report (2020, p. 2) also included the following recommendation:

Extensive societal dialogue should be undertaken before a country makes a decision on whether to permit clinical use of heritable human genome editing (HHGE). The clinical use of HHGE raises not only scientific and medical considerations, but also societal and ethical issues that were beyond the Commission's charge. 
There are prior examples of societal upheaval resulting from the exclusion of major segments of the population in making decisions that influence their lives. With HHGE unleashed toward genome improvement, combined and interacting with AI, continuous societal upheaval caused by intergenerational conflicts and struggles between adopters and non-adopters of both GM and AI can be expected. We can expect due to the adoption of competing designs, and change merely through rapid evolution over time, societal disparities to emerge between "organics" and "synthetics." Those who can and do move to a new adaptive landscape leaving their ancestral relatives to their much slower, and different, organic evolutionary path may become increasingly influential toward the normalization of eugenics.

\section{Social Pressures on New "Transhumanist Norms"}

The mere recognition that social pressures may influence the adoption of these technologies will not prevent such cultural shifts in norms. Demand for one-time treatment with genetic "medication" that could confer immunity to pathogens (socalled "DNA vaccines"), for example, could lead to factions in society, including schisms among scientists and friction among nations with different moral landscapes. The results presented in this study suggest that humans adopting genetic modification and AI augmentation can be expected to diverge from non-adopters at roughly a two-to-three-fold pace, potentially greater, as they acquire advances in hardware and software. A two-class system would quickly emerge. Recent trends in health choices foreshadow a future in which societal pressures to conform to adoption of emerging realm-creating technology manifest (e.g., pressure to prepare one's child by kindergarten for calculus). In some nations, it can be expected that laws might be passed mandating genetic modification, or surgery for brain implants, or mandating an hour of use of something like transcranial magnetic stimulation plus soundwave inputs of information into the brains of our youth so they can be "school ready" or "able to integrate" into an increasingly modified society. "Did you get the latest upgrade?" may become commonplace discussion about one's own brain. Resumes may include specs similar to those for computers. Increasing pressure due to international competition for economic dominance can be expected to be offered as patriotic justification for enforced adoption. Given the unknown outcomes, the decision to lock in a fate of eternal updates in CP, accelerating at everincreasing rates due to compounding phenotypes of endogenous origin, would seem to be a grave decision of massive consequence for all of humanity.

Given the curves our functions imply, our limited ability to predict the unintended consequences of such an emergence must be met with priority attention to issues of regulation, marketing, and widespread general use by the public. There is a hyper-bandwagon of direct manipulation of human evolution coming. Clearly, at some time, part of the evolution of our consciousness must involve a considered and specific decision on whether we should make that jump.

\section{Biological Class Structures and the Genetic and Economic Elite}

If market forces drive the production and availability of emerging biotransformative technologies, which is nearly a certainty, then those with resources to acquire and use the technology will ride the runaway train to ever-increasing inherited abilities and, it is safe to presume, ever-increasing wealth. Their continued demand for ever-increasing abilities to compete with other adopters will likely fuel the runaway evolution, leaving those without resources for self-modification in poverty. The disparity between the "haves" and the "have-nots" will become greater, leading to increased likelihood of the economic manipulation of the second-class citizenry by the genetic and economic elite.

\section{Standing Up for "Transhumanist" Animals}

In principle, there is nothing stopping the application of CP GM and AI technologies to animals. Given the massive increase in the rate of $\mathrm{CP}$ evolution made possible by these factors, it is theoretically possible that we will face the ethical question of whether we should allow them to be used on animals for curiosity, for entertainment (e.g., cats that "glow in the dark" because humans enhanced their genome with green fluorescent proteins), or for the exploration of interspecies relationships. Each evolutionary lineage arguably has its own right to an evolutionary future not determined so unilaterally and so strongly by human-derived "design feature" technologies.

\section{Limitations}

Our analysis does not distinguish between vertical genetic and cultural transmission of modification of traits. This is in part because while the genetic modification of the soma may not be inherited, the tendency of individuals to similarly modify their offspring is assumed. Current considerations on allowing somatic genetic manipulations must take this fact into consideration. Similarly, the adoption of direct human-computer interfaces can be expected to be shared within families. Our crystal ball is imperfect; i.e., we cannot anticipate other forces that might override those that we address - for example, catastrophic asteroid impacts that return us materially to the Stone Age (if some of us were 
to survive) - but the same principles that we have explored would be expected to apply with a reemergence of a society capable of technological advances.

\section{Conclusions}

In a very sober and materially significant way, the unknown emergences due to PEOs can be expected to seem foreign, rapid, confusing, and socially divisive in ways that have been mostly unanticipated and not completely imagined in scope. Environmental factors of the fourth, and by extension fifth kind represent uncontrolled self-evolutionary experiments that, like programmed eugenics, should be considered a threat to egalitarian views of humanity. The "singularity" predicted by Kurzweil in which artificial intelligences surpass humans and take over their own design has, according to Elon Musk, a definitive answer: Musk has stated that he thinks we should "merge with them" (CNBC 2017). I think we should have a good, hard think first: the adoption solution only shifts the competition between adopters and non-adopters; augmented humans versus non-augmented humans, and the resulting potential for societal upheaval requires deep contemplation by all interested parties; by definition, we are all impacted by which evolutionary landscape humanity steps upon, whether we participate as individuals or not.

On the issue of heritable genetic modifications, opinions range from outright bans (Newman 2019), through moratorium (e.g., Lander et al. 2019), to proponents who assign themselves the role of speaking for the children who might not be born, or who might be born with serious conditions were it not for heritable genetic modifications (Kleideman 2019; Knoppers et al. 2019). The position on outright banning the introduction or modification of heritable variation is, according to Newman (2019), to prevent complete "dissolution" of the species. The position of moratorium implies a temporary forbearance until the inevitable adoption and creates a potentially steep slope. Even bans on genetic modification, as seen with the "ban" on gain-of-function research, is a leaky barrier in a complex, competitive international climate. The position in favor of heritable genetic modifications is counterbalanced by recognition of the failure for deep appreciation of the complexity of human developmental genetics (Rossant 2018), which involves epistasis, pleiotropy, gene $\mathrm{x}$ environment interactions, and the concern of off-target effects (Horton and Lucassen 2019), the effects of which cannot be known perhaps for an entire generation or two.

The issue has raised considerations ranging from the prescient treatment of the genetic modification of behavior, personality, and psychology by Neitzke (2012) to focus on differences in the nature of parenting as vertical transmission and the alteration of children by parents and contemplation on the nature of intergenerational social contracts (Rehmann-Sutter 2018). Since the myopia of biomedicine does not often yield elegantly to balance of risk considerations, these issues must, ultimately, be decided by sectors of society not involved in direct or indirect financial gain from adoption. Darnovsky and Hasson (2020) correctly call for cultural input from sectors of our societies far removed from science; Chan (2020) has called for global regulation, but by means unknown. I would add that those who stand to financially benefit be excluded from debates on the moral questions around adopting biotransformative technologies.

Another aspect of modification of humans is the development of chimeric individuals; as multicellular organisms, such experiments have already been conducted, resulting in rat/mouse, sheep/goat, mouse/human, pig/human, and most recently monkey/human chimeric embryos (Tan et al. 2021).

Bringing chimeras involving human stem cells to term has generally been considered unethical for a number of reasons, including issues ranging from zoonoses from retroviral transfer among species to farming animals with human tissues (Bourret et al. 2016). Experiments in China leading to human/monkey chimeras have been met with raised eyebrows and limited discourse on ethics (Greely and Farahany 2021; NAS 2021). So far, the plan appears to be to state functional limits on how long human/animal chimeric embryos are allowed to develop (to date 14 days, before neurological development), and then relax them (Regalado 2021).

To the extent that adult-stage organ transplant leads to chimerism, it has also been conducted at the adult level (e.g., pig hearts into human patients), with little overt regard for the likelihood of zoonotic transfer of viruses, a topic not addressed or anticipated by those conducting experiments leading to chimeric embryos.

Genetic modification would likely lead to runaway competition in desired abilities; for example, concern over the ease of "gene doping" in sports is being addressed with a search for means to detect it and enforce a ban on such practices. There are an increasing number of technologies for genetic manipulation as well as an increasing number of genes that are considered likely candidates for gene doping (see Cantelmo et al. 2020). This also leads to the concern over genetic modification of human soldiers to increase strength, stamina, endurance, risk tolerance, and other characteristics a despotic regime might foolishly believe are desirable. Societies around the globe must curtail military exploitations of biotransformative technologies and view the development of super soldiers as crimes against humanity.

Another likely area of application of genetic modification is life extension; incredibly, clinical trials of human experiments designed to extend telomeres (the capped ends of chromosomes that shorten as we age) is underway, with patients enrolling under an ethically dubious process 
requiring they pay a fee of $\$ 1$ million (Libella Gene Therapeutics 2019). Longer-lived humans who can genetically modify themselves to acquire new characteristics will likely initially incur high risks of off-target genomic modifications, leading to cancer or activation of silenced endogenous viruses. In terms of impact on the rate of evolution of our species, we only need imagine if your great-great-grandparents were still alive, with males potentially contributing to the gene pool. Prior generations' genetic diversity, including higher de novo germline mutations that accompany aging, could be expected to contribute to the total available genetic diversity, further lowering the limits of the rates of evolution. Truly long-lived individuals would of course lead to stagnation of evolution; in the extreme example of widespread sterility, the rate of evolution would be limited to the industry rates of "advances" in genetic modification and external cognition, and availability and cost of "upgrades."

All of these other technological modifications compound and diversify the fitness landscape with their own potential for impact on reaction norms but also as a potential source of new hyperrealms. The current analysis implies that the human species is still in its technological infancy; it is well worth remembering that we progressed from wood-burning steam engines to plasma drive engines in less than 250 years. There exists a diversity of opinion and awareness of genomic editing issues in medicine (Delhove et al. 2020). We cannot risk erring on the side of hubris under the illusion we can control all elements of society in all nations from adopting these new technologies in overt or diffuse breeding programs. The distinctions between providing protection from disease and "enhancement" and "enhancement" and "unfair advantage" are blurry (So et al. 2017) and together represent a slippery, alluring slope for some (Harris et al. 2015; Knoppers et al. 2019; Macintosh et al. 2019; Pompei et al. 2019; Isa et al. 2020; Savulescu and Singer 2019; Segers et al. 2020) and a moral mandate for others (e.g., Gyngell et al. 2019 and Kleiderman et al. 2019, who argue for a "right to science" and a "right to the highest attainable health").

Others see the issue of both bioengineering humans via genes and fusion with machines more clearly as science racing ahead of any moral framework (e.g., Marinelli and Del Rio 2020). The current state of non-enforceable moratoria that "allow" exploration of the clinical use of nonheritable genetic modifications and research on heritable genetic modifications and calls for inclusion of the public in such considerations sets the stage for the normalization of technology whose effects span generations, going well beyond the ability of standard paradigms of safety trials in human subjects research (Cwik 2020).

Halpern et al. (2019) call for a "human rights impact statement" approach to dealing with adoption or non-adoption of specific heritable genetic modifications. My analysis shows that such an approach does not go far enough to prevent externalization of costs of the unintended or indirect consequences: the primary consideration must be focused on the emergence of hyperrealms and hastened biological and sociological differences between sectors of society. There are other options, such as passing laws regulating the definitive rights of humans to have say over their own bodies, an "ultimate genomic autonomy bill of rights," and developing norms that disallow the intentional or unintentional alteration of human evolution by genetic modification or genetic augmentation technologies. The seeming inevitability of adoption of heritable GMs by illicit means, or in countries that choose to progress regardless of the exponential effects, is neither a sufficient reason nor an excuse for doing so. We must undergo no faulty logic on the path to the future we must share. Flawed moral arguments that will likely be made are proven tactics, and include the creation of the perception of demand, presentation of an egalitarian argument that ensuring access to underprivileged population is a moral imperative, or getting the government to pay for it via mandates. Given the potential costs to our evolutionary future, we must never, for example, pathologize dissent.

The reliance on arguments of absence of evidence of risk two generations before it can be manifest will be recalled as bad genomic and evolutionary stewardship. I therefore urge an immediate moratorium on any human experimentation that has the capacity to alter permanently the adaptive landscape upon which we were born in a manner that can establish a new hyperrealm. The evolutionary landscape upon which we were born is the most basic and universal of all common rights, upon which all our descendants, modified and augmented or not, must and are entitled to live free from manipulation of recently emerged Homo sapiens who do not even yet know how to run pluralistic societies without

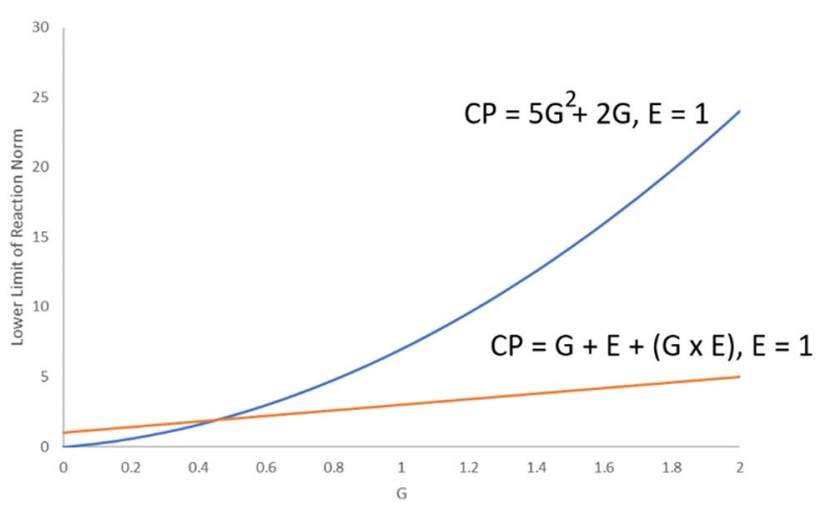

Fig. 4 The two functions of primary interest compared, with fixed value $\mathrm{E}=1$. The initial manipulations appeal, given the reduction of the relative impact of seemingly uncontrolled $\mathrm{E}$ being overtaken by endogenous self-manipulation; the resulting exponent resulting from the hubris of adoption leads (minimally) to ever-increasing lower limits of reaction norms with paradoxically no influence of E. Note the initial state of $G+E+(G x E)>5 G^{2}+2 G$ may prevent the awareness of the increased rate to come with additional introduced modifications 
violence. We have a collective right and responsibility to take an extremely cautious approach toward modifying the adaptive landscape upon which we all, and our progeny, as a species have no choice but to continue to collectively evolve.

\section{Appendix}

To show where $5 G^{2}+2 G$ diverges from $C P=G+E+(G x$ $E$ ), both functions are plotted using a fixed value of $E=1$, allowing $G$ to vary. In this representation, $G$ includes the degree of endogenous impact of a species on the lower limit of the reaction norm. The obsolescence of exogenous $E$ is made obvious with increasing impact of $G$ on the genetic variation of endogenous origin. The same relative function placements persist with variation in $E$ for all $E>0$ but as $E$ is increased (see Fig. 4).

Acknowledgments The author and readers collectively have the entire body of theoretical and empirical evolutionary research to thank for the cornerstones upon which this attempt to communicate a most dire warning is founded, and thus to the authors of the citations and those who have contributed massively to evolutionary biology and their mentors, I offer my gratitude. I thank Garth Stein for his enduring curiosity for the bizarre and uncanny, and for challenging me to attempt to write a paragraph he might understand, and for his numerous other candid remarks. I am grateful to Guy Hoelzer for inspired discussions and remembrances of those who have contributed. To those who may dare to read morality into this work, I challenge all to a new body of theory predicated on the hypothesis that value-statement-free comprehension work might lead to insights on finding a moral basis for means by which a human civilization might prove effective and maintain intrinsic worth, and I thank them profusely for metacognitive efforts to eschew the opposite inference. At the present time, the perils of drawing lessons on society from biology seem a distant threat given the overwhelming potential threats of the effects of technology on biology, and thereby, on society.

Funding This analysis was funded by donations from the public to The Institute for Pure and Applied Knowledge.

Open Access This article is licensed under a Creative Commons Attribution 4.0 International License, which permits use, sharing, adaptation, distribution and reproduction in any medium or format, as long as you give appropriate credit to the original author(s) and the source, provide a link to the Creative Commons licence, and indicate if changes were made. The images or other third party material in this article are included in the article's Creative Commons licence, unless indicated otherwise in a credit line to the material. If material is not included in the article's Creative Commons licence and your intended use is not permitted by statutory regulation or exceeds the permitted use, you will need to obtain permission directly from the copyright holder. To view a copy of this licence, visit http://creativecommons.org/licenses/by/4.0/.

\section{References}

Assary E, Vincent JP, Keers R, Pluess M (2018) Gene-environment interaction and psychiatric disorders: review and future directions.
Semin Cell Dev Biol 77:133-143. https://doi.org/10.1016/j.semcdb.2017.10.016

Balcombe J (2016) Einstein of the sea. Sci Am 314(6):66-69. https:// doi.org/10.1038/scientificamerican0616-66

Beltran T, Shahrezaei V, Katju V, Sarkies P (2020) Epimutations driven by small RNAs arise frequently but most have limited duration in Caenorhabditis elegans. Nat Ecol Evol. https://doi.org/10.1038/ s41559-020-01293-Z

Bourret R, Martinez E, Vialla F, Giquel C, Thonnat-Marin A, De Vos J (2016) Human-animal chimeras: ethical issues about farming chimeric animals bearing human organs. Stem Cell Res Ther 7(1):87. https://doi.org/10.1186/s13287-016-0345-9

Burls KJ, Shapiro J, Forister ML, Hoelzer GA (2014) A nonlinear relationship between genetic diversity and productivity in a polyphagous seed beetle. Oecologia 175(1):151-161. https://doi.org/ 10.1007/s00442-014-2893-y

Cantelmo RA, da Silva AP, Mendes-Junior CT, Dorta DJ (2020) Gene doping: present and future. Eur J Sport Sci 20(8):1093-1101. https://doi.org/10.1080/17461391.2019.1695952

Chan S (2020) Playing it safe? Precaution, risk, and responsibility in human genome editing. Perspect Biol Med 63(1):111-125. https:// doi.org/10.1353/pbm.2020.0009

CNBC (2017) Elon Musk: humans must merge with machines or become irrelevant in AI age. https://www.cnbc.com/2017/02/ 13/elon-musk-humans-merge-machines-cyborg-artificial-intel ligence-robots.html. Accessed 6 Nov 2020

Cross FM (1980) Newly found inscriptions in old Canaanite and early Phoenician scripts. Bull Am Sch Orient Res 238:1-20. JSTOR, www.jstor.org/stable/1356511. Accessed 25 Sep 2020.

Cwik B (2020) Intergenerational monitoring in clinical trials of germline gene editing. J Med Ethics 46(3):183-187. https://doi.org/ 10.1136/medethics-2019-105620

Darnovsky M, Hasson K (2020) CRISPR's twisted tales: clarifying misconceptions about heritable genome editing. Perspect Biol Med 63(1):155-176. https://doi.org/10.1353/pbm.2020.0012

De Marco RJ (2006) How bees tune their dancing according to their colony's nectar influx: re-examining the role of the food-receivers' 'eagerness.' J Exp Biol 209(3):421-432. https://doi.org/10. 1242/jeb.02025

De Marco R, Menzel R (2005) Encoding spatial information in the waggle dance. J Exp Biol 208:3885-3894

Delhove J, Osenk I, Prichard I, Donnelley M (2020) Public acceptability of gene therapy and gene editing for human use: a systematic review. Hum Gene Ther 31(1-2):20-46. https://doi.org/ 10.1089/hum.2019.197

Dobzhansky T (1970) Genetics of the evolutionary process. Columbia University Press, New York

Dudbridge F (2013) Power and predictive accuracy of polygenic risk scores. PLoS Genet https://doi.org/10.1371/annotation/b91ba 224-10be-409d-93f4-7423d502cba0. Erratum in: PLoS Genet https://doi.org/10.1371/annotation/b91ba224-10be-409d-93f4$7423 \mathrm{~d} 502 \mathrm{cba0}$

Ebrey PB (2010) The Cambridge illustrated history of China. Cambridge University Press, Cambridge

Greely HT (2019) CRISPR'd babies: human germline genome editing in the 'He Jiankui affair.' J Law Biosci 6(1):111-183. https://doi.org/10.1093/jlb/lsz010

Greely HT, Farahany NA (2021) Advancing the ethical dialogue about monkey/human chimeric embryos. Cell 184(8):19621963. https://doi.org/10.1016/j.cell.2021.03.044

Gyngell C, Bowman-Smart H, Savulescu J (2019) Moral reasons to edit the human genome: picking up from the Nuffield report. J Med Ethics 45(8):514-523. https://doi.org/10.1136/medet hics-2018-105084

Halpern J, O'Hara SE, Doxzen KW, Witkowsky LB, Owen AL (2019) Societal and ethical impacts of Germline genome 
editing: how can we secure human rights? CRISPR J 2(5):293298 https://doi.org/10.1089/crispr.2019.0042. Erratum in: CRISPR J 2(6):451. Witkowsy, Lea B [corrected to Witkowsky, Lea B]

Harris J (2015) Germline manipulation and our future worlds. Am J Bioeth 15(12):30-34. https://doi.org/10.1080/15265161.2015. 1104163

Harris K, Pritchard JK (2017) Rapid evolution of the human mutation spectrum. Elife 6:e24284

Henrique G (2019) Toward a metaphysical empirical psychology. Re-envisioning theoretical psychology, Palgrave studies in the theory and history of psychology (Palmgrave/Macmillan). Springer, Cham, pp 209-237

Horton R, Lucassen AM (2019) The moral argument for heritable genome editing requires an inappropriately deterministic view of genetics. J Med Ethics 45(8):526-527. https://doi.org/10. 1136/medethics-2019-105390

Isa NM, Zulkifli NA, Man S (2020) Islamic perspectives on CRISPR/ Cas9-mediated human germline gene editing: a preliminary discussion. Sci Eng Ethics 26(1):309-323. https://doi.org/10.1007/ s11948-019-00098-z

Joy MT, Ben Assayag E, Shabashov-Stone D, Liraz-Zaltsman S, Mazzitelli J, Arenas M et al (2019) CCR5 is a therapeutic target for recovery after stroke and traumatic brain injury. Cell 176(5):1143-1157.e13. https://doi.org/10.1016/j.cell.2019.01. 044

Kimura M (1983) The neutral theory of molecular evolution. Cambridge University Press, Cambridge

Kleiderman E, Ravitsky V, Knoppers BM (2019) The 'serious' factor in germline modification. J Med Ethics 45(8):508-513. https:// doi.org/10.1136/medethics-2019-105436

Knoppers BM, Kleiderman E (2019) Heritable genome editing: who speaks for 'future' children? CRISPR J 2(5):285-292. https:// doi.org/10.1089/crispr.2019.0019

Lander ES, Baylis F, Zhang F, Charpentier E, Berg P, Bourgain C et al (2019) Adopt a moratorium on heritable genome editing. Nature 567(7747):165-168. https://doi.org/10.1038/ d41586-019-00726-5

Le Rouzic A, Carlborg Ö (2008) Evolutionary potential of hidden genetic variation. Trends Ecol Evol 23(1):33-37

Lewontin R (1978) Adaptation. Sci Am 239:212-229

Libella Gene Therapeutics, LLC (2019) (Press Release) Breakthrough gene therapy clinical trial is the world's first that aims to reverse 20 years of aging in humans. https://www.prnew swire.com/news-releases/breakthrough-gene-therapy-clinicaltrial-is-the-worlds-first-that-aims-to-reverse-20-years-of-agingin-humans-300963496.html. Accessed 22 July 2021

Lynch M (2016) Mutation and human exceptionalism: our future genetic load. Genetics 202(3):869-875. https://doi.org/10.1534/ genetics.115.180471

Macintosh KL (2019) Heritable genome editing and the downsides of a global moratorium. CRISPR J 2(5):272-279. https://doi. org/10.1089/crispr.2019.0016

Marinelli S, Del Rio A (2020) Beginning of life ethics at the dawn of a new era of genome editing: are bioethical precepts and fast-evolving biotechnologies irreconcilable? Clin Ther 171(5):e407-e411

Moyers BT, Morrell PL, McKay JK (2018) Genetic costs of domestication and improvement. J Hered 109(2):103-116. https://doi. org/10.1093/jhered/esx069

National Academy of Sciences (2020) Heritable human genome editing, report summary. https://www.nap.edu/resource/25665/Herit able\%20Human\%20Genome\%20Editing\%20Report\%20Sum mary\%20-\%20FINAL\%2020200903.pdf. Accessed 28 Sep 2020
National Academy of Sciences (2021) The emerging field of human neural organoids, transplants and chimeras: science, ethics and governance consensus study report. https://www.nap.edu/catal og/26078/the-emerging-field-of-human-neural-organoids-trans plants-and-chimeras. Accessed 29 June 2021

Nei M, Rooney AP (2005) Concerted and birth-and-death evolution of multigene families. Annu Rev Genet 39:121-152. https://doi. org/10.1146/annurev.genet.39.073003.112240

Neitzke AB (2012) On the genetic modification of psychology, personality, and behavior. Kennedy Inst Ethics J 22(4):307-343

Newman SA (2019) Sex, lies, and genetic engineering: why we must (but won't) ban human embryo modification. In: Braverman I (ed) Gene editing, law and the environment: life beyond the human. Glasshouse. Routledge, New York. https://doi.org/10. 4324/9781315168418-8

Nieder A, Wagener L, Rinnert P (2020) A neural correlate of sensory consciousness in a corvid bird. Science 369(6511):1626-1629. https://doi.org/10.1126/science.abb1447

Noble DW, Carazo P, Whiting MJ (2012) Learning outdoors: male lizards show flexible spatial learning under semi-natural conditions. Biol Lett 8(6):946-948. https://doi.org/10.1098/rsbl. 2012.0813

Ohta T (1973) Slightly deleterious mutant substitutions in evolution. Nature 246(5428):96-98. https://doi.org/10.1038/246096a0

Ohta T (2002) Near-neutrality in evolution of genes and gene regulation. Proc Natl Acad Sci USA 99(25):16134-16137. https://doi. org/10.1073/pnas.252626899

Orr HA (1996) Dobzhansky, Bateson, and the genetics of speciation. Genetics 144(4):1331-1335

Petie R, Garm A, Hall MR (2016) Crown-of-thorns starfish have true image forming vision. Front Zool 13(1):41. https://doi.org/10. 1186/s12983-016-0174-9

Pigliucci M (2005) Evolution of phenotypic plasticity: where are we going now? Trends Ecol Evol 20(9):481-486

Pompei M, Pompei F (2019) Overcoming bioethical, legal, and hereditary barriers to mitochondrial replacement therapy in the USA. J Assist Reprod Genet 36(3):383-393. https://doi.org/10. 1007/s10815-018-1370-7

Regalado A (2021) Scientists plan to drop the 14-day embryo rule, a key limit on stem cell research. MIT Technology Review. https://www.technologyreview.com/2021/03/16/1020879/scien tists-14-day-limit-stem-cell-human-embryo-research/. Accessed 29 June 2021

Rehmann-Sutter C (2018) Why human germline editing is more problematic than selecting between embryos: ethically considering intergenerational relationships. New Bioeth 24(1):9-25. https://doi.org/10.1080/20502877.2018.1441669

Rossant J (2018) Gene editing in human development: ethical concerns and practical applications. Development 145(16):dev150888. https://doi.org/10.1242/dev.150888

Savulescu J, Singer P (2019) An ethical pathway for gene editing. Bioethics 33(2):221-222. https://doi.org/10.1111/bioe.12570.

Schlichting C, Pigliucci M (1998) Phenotypic evolution: a reaction norm perspective. Sinauer, Sunderland

Segers S, Mertes H (2020) Does human genome editing reinforce or violate human dignity? Bioethics 34(1):33-40. https://doi.org/ 10.1111/bioe. 12607

Shackleton K, Balfour NJ, Al Toufailia H, Gaioski R Jr, de Matos BM, Silva CAS et al (2016) Quality versus quantity: foraging decisions in the honeybee (Apis mellifera scutellata) feeding on wildflower nectar and fruit juice. Ecol Evol 6(19):7156-7165. https://doi.org/10.1002/ece3.2478

So D, Kleiderman E, Touré SB, Joly Y (2017) Disease resistance and the definition of genetic enhancement. Front Genet 10(8):40. https://doi.org/10.3389/fgene.2017.00040 
Stacho M, Herold C, Rook N, Wagner H, Axer M, Amunts K, Güntürkün O (2020) A cortex-like canonical circuit in the avian forebrain. Science 369(6511):eabc5534. https://doi.org/10.1126/ science.abc5534

Tan T, Wu J, Si C, Dai S, Zhang Y, Sun N et al (2021) Chimeric contribution of human extended pluripotent stem cells to monkey embryos ex vivo. Cell 184(8):2020-2032.e14. https://doi.org/10. 1016/j.cell.2021.03.020. Erratum in: Cell. 2021 184(13):3589

Van Valen L (1973) A new evolutionary law. Evolut Theory 1:1-30.

Wallace B (1953) On coadaptation in drosophila. Am Nat $87: 343-358$

WHO (2019) WHO launches global registry on human genome editing. World Health Organization, News. https://www.who. int/news/item/29-08-2019-who-launches-global-registry-onhuman-genome-editing. Accessed 29 June 2021

Wilkinson A, Huber L (2011) Cold-blooded cognition: reptilian cognitive abilities. In: Shackelford TK, Vonk J (eds) The Oxford handbook of comparative evolutionary psychology. Oxford University Press, New York

Wright S (1984) Evolution and the genetics of populations: genetics and biometric foundations new edition. University of Chicago Press, Chicago
Zhadan PM, Vaschenko MA (2019) Long-term study of behaviors of two cohabiting sea urchin species, Mesocentrotus nudus and Strongylocentrotus intermedius, under conditions of high food quantity and predation risk in situ. PeerJ 22(7):e8087. https:// doi.org/10.7717/peerj.8087

Zhang F, Zhai HQ, Paterson AH, Xu JL, Gao YM, Zheng TQ, Wu RL, Fu BY, Ali J, Li ZK (2011) Dissecting genetic networks underlying complex phenotypes: the theoretical framework. PLoS ONE 6(1):e14541. https://doi.org/10.1371/journal.pone. 0014541

Zhou M, Greenhill S, Huang S, Silva TK, Sano Y, Wu S et al (2016) CCR5 is a suppressor for cortical plasticity and hippocampal learning and memory. Elife 20(5):e20985. https://doi.org/10. 7554/eLife

Publisher's Note Springer Nature remains neutral with regard to jurisdictional claims in published maps and institutional affiliations. 